\title{
Personalized Mechanotherapy: Simultaneous Correction of Posterior Crossbite and Anterior Open Bite in the Presence of Root Shortening
}

\author{
Abdullah $\mathrm{F}^{\mathrm{a}}$, Sangsuwon $\mathrm{C}{ }^{\mathrm{a}}$, Alansari $\mathrm{S}^{\mathrm{a}}$
}

a CTOR Academy, Hoboken, New Jersey

Corresponding Author:

Sarah Alansari

sarah.alansari@ctor.clinic

Citation:Abdullah F, Sangsuwon C, Alansari S. Personalized mechanotherapy: simultaneous correction of posterior crossbite and anterior open bite in the presence of root shortening. Innovation. 2020. 3(1):e.1. doi:10.30771/2020.1.

Submitted December 10, 2020

Accepted December 15, 2020

Keywords: Personalized; Mechanotherapy; Orthodontics; Posterior crossbite; Anterior open bite; Root resorption

\section{ABSTRACT}

The majority of Orthodontics patients have a wide variety of problems that challenge the clinician to design a personalized mechanotherapy based on each patient's specific needs. This customized mechanotherapy should address the patient's problems efficiently to not only minimize treatment duration but also reduce the risk or potential side effects associated with unnecessary movement or prolonged treatment. Equally important, a customized mechanotherapy plan educates the patient about benefits and risks of different treatment options, and engages them during treatment as they observe the changes predicted in their specific treatment plan. In this article we present a personalized mechanotherapy plan and its outcome in the context of an Orthodontic retreatment for a patient with an open bite, crossbite, root resorption, and previously extracted four premolars. 


\section{Background}

Many adults suffer from open bites that range from mild to severe. When the open bite is limited to the anterior teeth, and particularly when these teeth are proclined, a simple treatment approach such as the retraction of the anterior teeth may be sufficient to correct the open bite. With this approach in mind, it is common for clinicians to extract upper and lower 1st premolars in an attempt to create space for retracting upper and lower anterior teeth [1]. But what happens if the open bite extended to the posterior teeth, which cannot be addressed by simply uprighting anterior teeth? And what if the patient has had previous Orthodontic treatment and the attempt to correct the open bite by four premolars extraction had already failed?

Another challenging factor in some open bite cases is the simultaneous presence of a posterior crossbite. This can be discouraging since the correction of a posterior crossbite, regardless of the methodology chosen, can be accompanied with bite opening and therefore worsening of the anterior open bite [2].

An additional factor that can significantly contribute to the complexity of treatment is the presence of root resorption or root blunting, which may or may not be related to the previous orthodontic treatment [3]. Should we even consider orthodontic retreatment for these patients, knowing the possibility that root resorption may increase? This is especially a concern when it is the anterior teeth that have undergone root resorption, as significant movement of these teeth is required to resolve the open bite.

Here we present a patient with a complex, challenging malocclusion: anterior open bite, anterior and posterior crossbites, anterior root resorption and previous extraction of the maxillary $2^{\text {nd }}$ and mandibular $1^{\text {st }}$ premolars. The most distal portion of the patient's posterior crossbite was due to four fully erupted $3^{\text {rd }}$ molars, which she refused to have extracted. In addition, since the patient had undergone orthodontic treatment in the past, she was not very keen on the idea of long treatment with fixed appliances (braces) and she requested a short treatment period.

All of these difficulties contribute to the complexity of this case and demand a personalized, carefully staged mechanotherapy plan.

\section{Patient Presentation, Etiology and Diagnosis}

A 29.8-year-old hypertensive female patient presented to the clinics of CTOR Academy with a chief concern of crossbite and open bite. The patient reported that she had a grinding habit but no history of trauma or signs or symptoms of temporomandibular joint disorder. During her first orthodontic treatment 13 years earlier, four premolars were extracted. However, treatment had failed to fully correct the crossbite and open bite.

Extraoral examination (Figure 1) and frontal portrait photographs show a mesiofacial pattern. The lower third of the face was normal, and the lips were competent. Facial asymmetry was noted as a slight deviation of the mandible to the right side.

The lateral portrait photograph shows a straight profile and a slightly increased nasolabial angle and chin-to-throat angle $\left(100^{\circ}\right.$ and $132^{\circ}$, respectively). The upper and lower lips were deficient according to the E-line distance. Upon smiling, the patient revealed a straight smile line with $80 \%$ incisal display.

Clinical intraoral examination (Figure 1) showed good oral hygiene, normal frenum attachment, and noticeable bilateral mandibular tori. Digital cast analysis showed a Class III canine and molar relationship on the right side, and a Class I canine and molar relationship on the left side. Crossbites were observed on the right lateral incisor, canine, premolars and third molars. Mild crowding was evident on the lower incisors $(3.3 \mathrm{~mm})$ and wear was noted on several cusps, supporting a grinding habit. The maxillary 2nd premolars and the mandibular 1st premolars, were previously extracted. The patient had an overjet ranging from $3.2 \mathrm{~mm}$ to $-1.1 \mathrm{~mm}$, and an overbite ranging from $0.3 \mathrm{~mm}$ to $-1.5 \mathrm{~mm}$. A Bolton discrepancy was also noticed due to maxillary deficiency of upper anterior teeth $(3.4 \mathrm{~mm})$.

Panoramic (Figure 2) and lateral cephalometric (Figure 3) radiographs were taken 1 week prior to the beginning of treatment. The panoramic radiograph showed mild to severe root resorption on several teeth in the anterior and premolar areas. Lateral cephalometric (Figure 3 ) analysis (Table 1) showed a Class I skeletal relationship (ANB $=4.0^{\circ}$ ) with a normo-divergent profile (FMA $\left.=24.9^{\circ}\right)$. The maxillary incisor inclination was increased $\left(\mathrm{U} 1^{\circ}\right.$ with $\left.\mathrm{SN}=112.9^{\circ}\right)$, while the mandibular incisor inclination was within the normal range $\left(\mathrm{IMPA}=94.3^{\circ}\right)$. Soft tissue analysis $($ Table 1$)$ indicated that the distance of the upper and lower lips to E-line was deficient, with measurements of $-6.3 \mathrm{~mm}$ and $-3.0 \mathrm{~mm}$, respectively. 

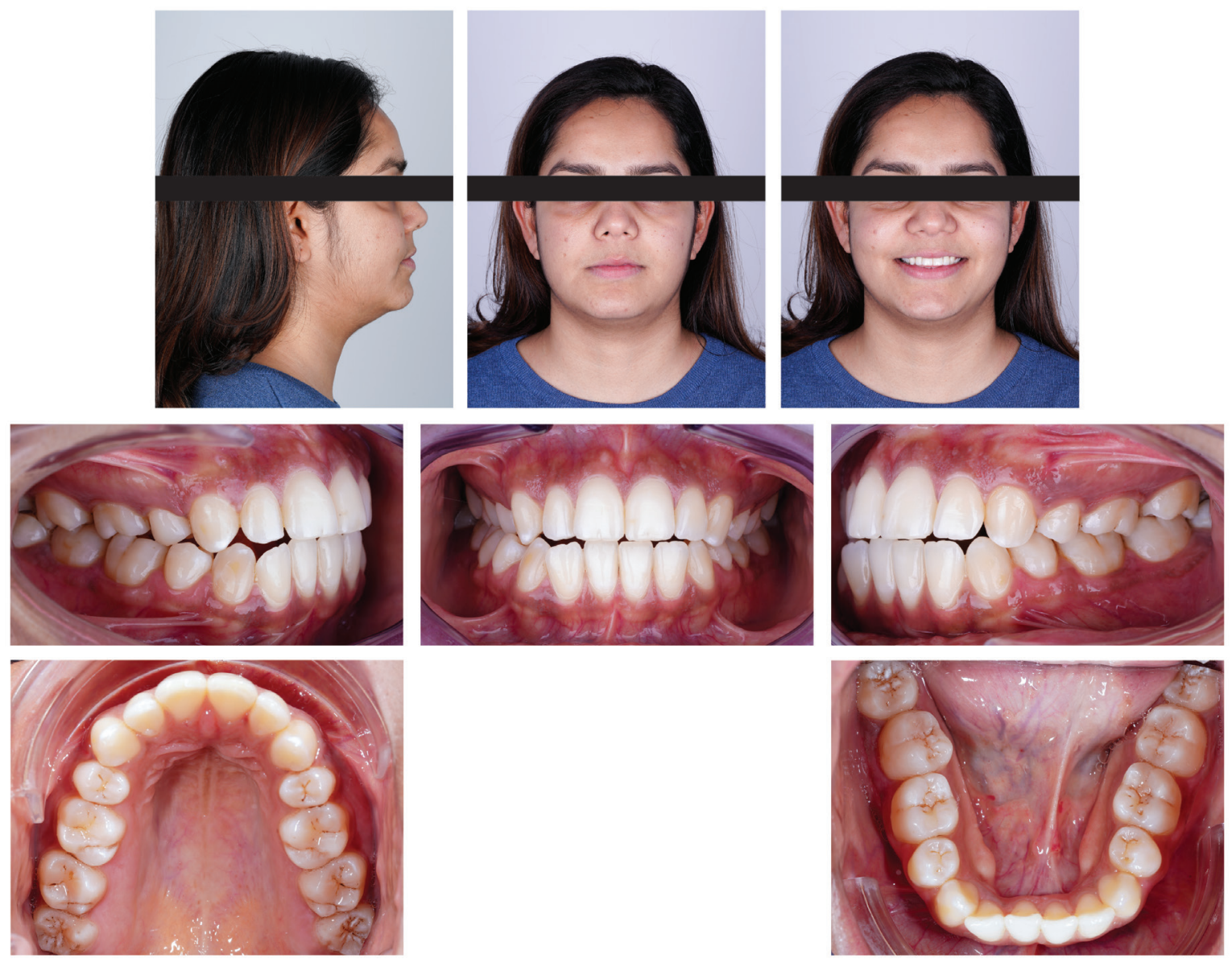

Figure 1: Pretreatment extra- and intra-oral photographs. Portrait photographs before initiation of treatment show a straight profile, slightly asymmetrical face due to mandibular deviation to the right side, dark buccal corridors an 80\% incisal display while smiling. Intraoral photographs reveal a constricted maxilla, anterior open bite, anterior and posterior crossbites, crowding and proclined maxillary anterior teeth. Wear facets can be observed in several teeth suggesting a gridding habit.

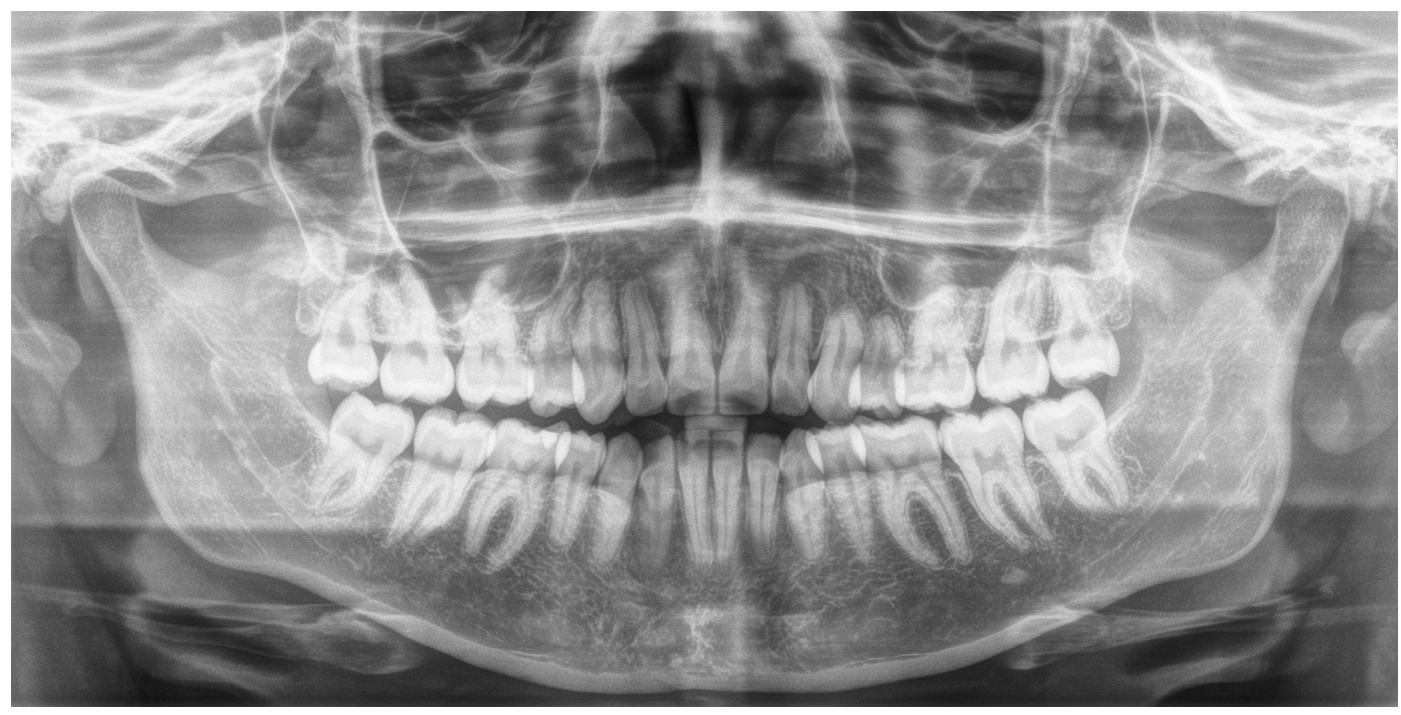

Figure 2: Pretreatment panoramic radiograph. Panoramic radiograph shows a complete dentition except for the absence of upper second and lower first premolars, spindle-like roots on molars, mild to severe root resorption of the maxillary central incisors, canines and premolar, and the lower right canine and premolar. Asymmetrical condyles show some degree of remodeling and asymmetrical position. Panoramic radiography showed an area of idiopathic sclerosis close to the root of the second left molar. 

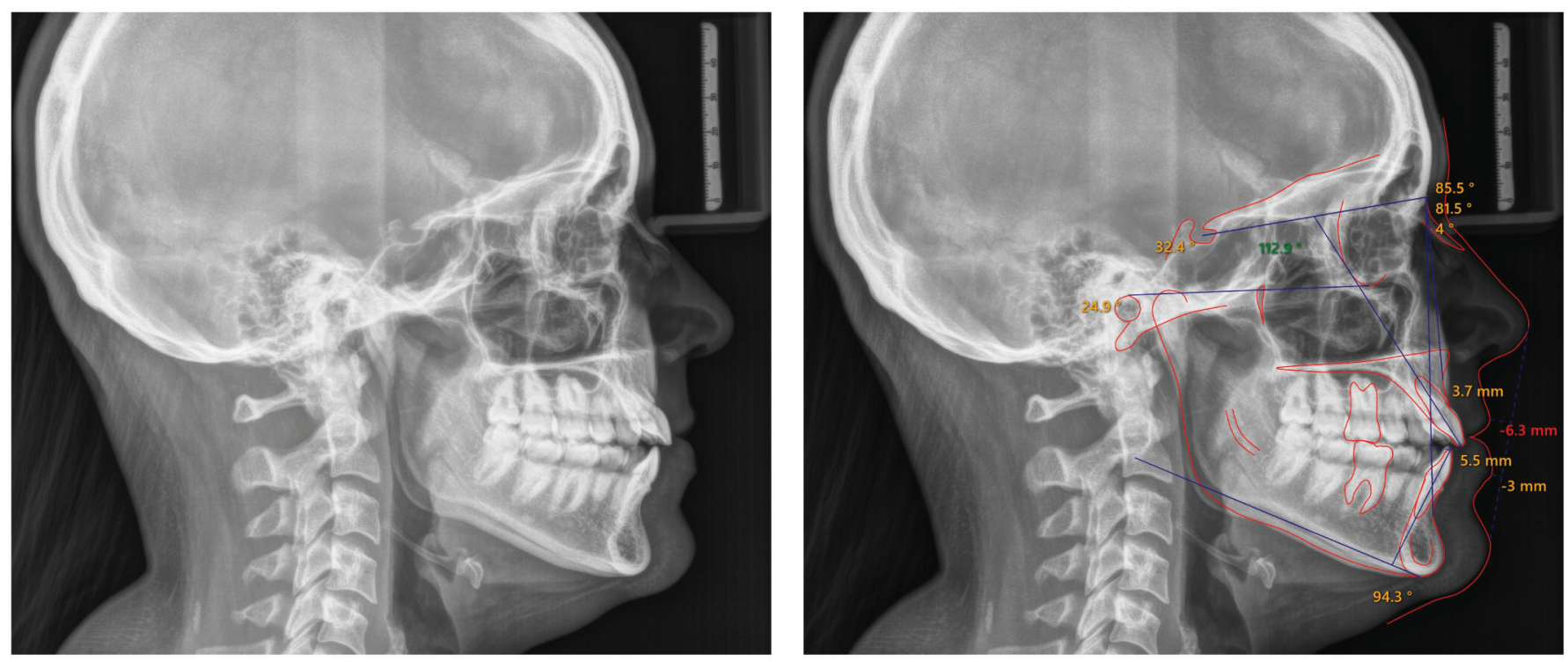

Figure 3: Pretreatment lateral cephalometric radiograph and analysis. Cephalometric analysis clearly shows the anterior open bite, class I skeletal relation of maxilla and mandible, counter-clockwise rotation of the maxillary occlusal plane, normal inclination of the mandibular plane, and proclined upper incisors.

\section{Measurement}

Upper Incisor Angle with SN

$$
\text { SNA Angle }
$$

SNB Angle

ANB Angle

IMPA

FMA

SN-MP

Upper Incisor to NA mm

Lower Incisor to NA mm

Upper Lip to E-plane

Lower Lip to E-plane
Initial Value

$112.9^{\circ}$

$85.5^{\circ}$

$81.5^{\circ}$

$4.0^{\circ}$

$94.3^{\circ}$

$24.9^{\circ}$

$32.4^{\circ}$

$3.7 \mathrm{~mm}$

$5.5 \mathrm{~mm}$

$-6.3 \mathrm{~mm}$

$-3.0 \mathrm{~mm}$

Table 1: Cephalometric Analysis Pre- and Posttreatment
Final Value

Norm

$106.9^{\circ} \quad 102.4 \pm 5.5^{\circ}$

$85.2^{\circ}$

$81.8 \pm 3.7^{\circ}$

$81.7^{\circ}$

$79.2 \pm 2.3^{\circ}$

$3.5^{\circ}$

$2.6 \pm 2.4^{\circ}$

$92.3^{\circ}$

$92.1 \pm 9^{\circ}$

$26.6^{\circ}$

$25.8 \pm 3^{\circ}$

$31.2 \pm 3^{\circ}$

$34^{\circ}$

$3.8 \pm 2.7 \mathrm{~mm}$

$6.7 \mathrm{~mm}$

$3.4 \pm 3.6 \mathrm{~mm}$

$-5.5 \mathrm{~mm}$

$-2 \pm 2 m m$

$-3.2 \mathrm{~mm}$

$-2 \pm 2 m m$ 


\section{Treatment Objective}

The overall treatment objective was to provide the patient with a normal functional occlusion and improved smile esthetics. The following specific objectives were planned:

I. Facial objectives: Improve the mandibular asymmetry and upper lip position relative to the E-line, and maintain the facial profile. II. Skeletal objectives: Improve the transverse relation, but maintain maxilla-to-mandible sagittal and vertical relations.

III. Dental objectives: Expand the maxillary arch, resolve the crowding, correct the mandibular midline deviation and obtain proper overjet and overbite relation without extracting any additional teeth.

IV. Maintain root length by minimizing the possibility of further root resorption.

\section{Treatment Options}

Patient was presented with different treatment options. Surgical options to address the skeletal problems were rejected. Since patient had braces before, she expressed interested in clear aligners as the treatment of choice due to the improved esthetics of these appliances. The limitations of this approach in addressing crossbite and open bite problems were discussed. Patient decided to proceed with a palatal expander and fixed appliances as long as treatment duration was reduced. To address the esthetic concern of the patient in wearing fixed appliances, we planned the use of sectional mechanics on posterior teeth initially, and only use braces on the anterior teeth later in treatment.

\section{Applied Innovation: Personalized Mecahnotherapy Plan}

Expansion and development of the maxilla was performed using a hyrax palatal expander, with bands on the first molars and arms extended anteriorly to the canines and posteriorly to the $3^{\text {rd }}$ molars. The patient was instructed to turn the expander once every three days. This regimen gradually increased to every other day until adequate expansion was achieved. After expansion was completed, a modified TPA was delivered to preserve the expansion and correct the inclination of $2^{\text {nd }}$ and $3^{\text {rd }}$ molars using a free object mechanical design [5]. Sectional fixed appliances were bonded on the posterior segments of upper and lower arches, including molar tubes on the $2^{\text {nd }}$ and $3^{\text {rd }}$ molars and self-ligating ceramic brackets on the premolars and canines. As treatment progressed, fixed appliances were bonded on the anterior teeth and a one-couple mechanical design was used for extrusion of upper anterior teeth. Meanwhile, a two-couple sectional mechanics in the $2^{\text {nd }}$ order view was applied to upright the posterior teeth [6]. These mechanics were supported with the use of selective elastic bands. Alikhani Bite-Block Therapy (ABBT) was applied to address the mandibular shift based on CNS (Central Nervous System) theory [5]. Coordination of the arches, finishing and detailing was accomplished with continuous arch wires. After 10 months of treatment, all appliances were removed, fixed retainers were bonded on upper and lower anterior teeth, and removable retainers with posterior occlusal coverage were provided to secure the stability of both arches.

\section{Treatment Outcome}

After only 10 months of treatment, all treatment objectives were achieved by customized and modified mechanics for precise movements and accelerated orthodontic treatment. The changes produced were the result of the dentoalveolar changes planned as treatment goals for this patient:

\section{Facial Objectives (Figure 4):}

A) At rest, the mandibular deviation was corrected.

B) Profile analysis demonstrated improvement in upper and lower lip position relative to the E-line.

C) Smile analysis showed a wider maxilla and significant decrease of the buccal corridors. Smile arch and incisal display were also significantly improved.

\section{Dental Objectives (Figure 4):}

A) Expansion and development of the maxillary arch (intermolar width increased by $2 \mathrm{~mm}$, while intercanine width increased by $3.2 \mathrm{~mm}$ ). This was accompanied by correction of crossbites, which was one of the patient's chief concern.

B) Crowding in both maxillary and mandibular arches was resolved and the proclination of maxillary incisors decreased.

C) Both maxillary and mandibular dental midlines were aligned with the facial midline.

D) Class I canine and molar occlusion was established. 
E) Open bite, another patient's major concern, was corrected through:

1. Extrusion and slight uprighting of maxillary anterior teeth, and extrusion of mandibular anterior teeth.

2. Uprighting of posterior teeth. These movements significantly helped flatten the Curve of Spee.

F) Gingival esthetic analysis showed that the gingival margins were leveled and the gingival height of contour was properly positioned around the anterior teeth.

G) Posttreatment panoramic radiograph (Figure 5) showed good root alignment with no additional root resorption.

\section{Skeletal Objectives and lateral cephalometric analysis (Figure 6):}

A) The posttreatment lateral cephalometric analysis (Table 1; Figure 6) showed that the Class I skeletal relationship was improved $\left(\mathrm{ANB}=3.5^{\circ}\right)$.

B) The maxillary incisor inclination was improved $\left(\mathrm{U} 1^{\circ}\right.$ with $\left.\mathrm{SN}=106.9^{\circ}\right)$

C) Superimposition of mandibular incisors did not demonstrate any increase in inclination, but slight extrusion.

D) Superimposition of the radiographs taken before and after treatment (Figure 7) showed that the maxillary and mandibular incisors extruded, and all 1st molars were uprighted.

E) Soft tissue analysis indicated that the distance of the upper and lower lips to E-line was slightly improved $(-5.5 \mathrm{~mm}$, $-3.2 \mathrm{~mm}$, respectively).
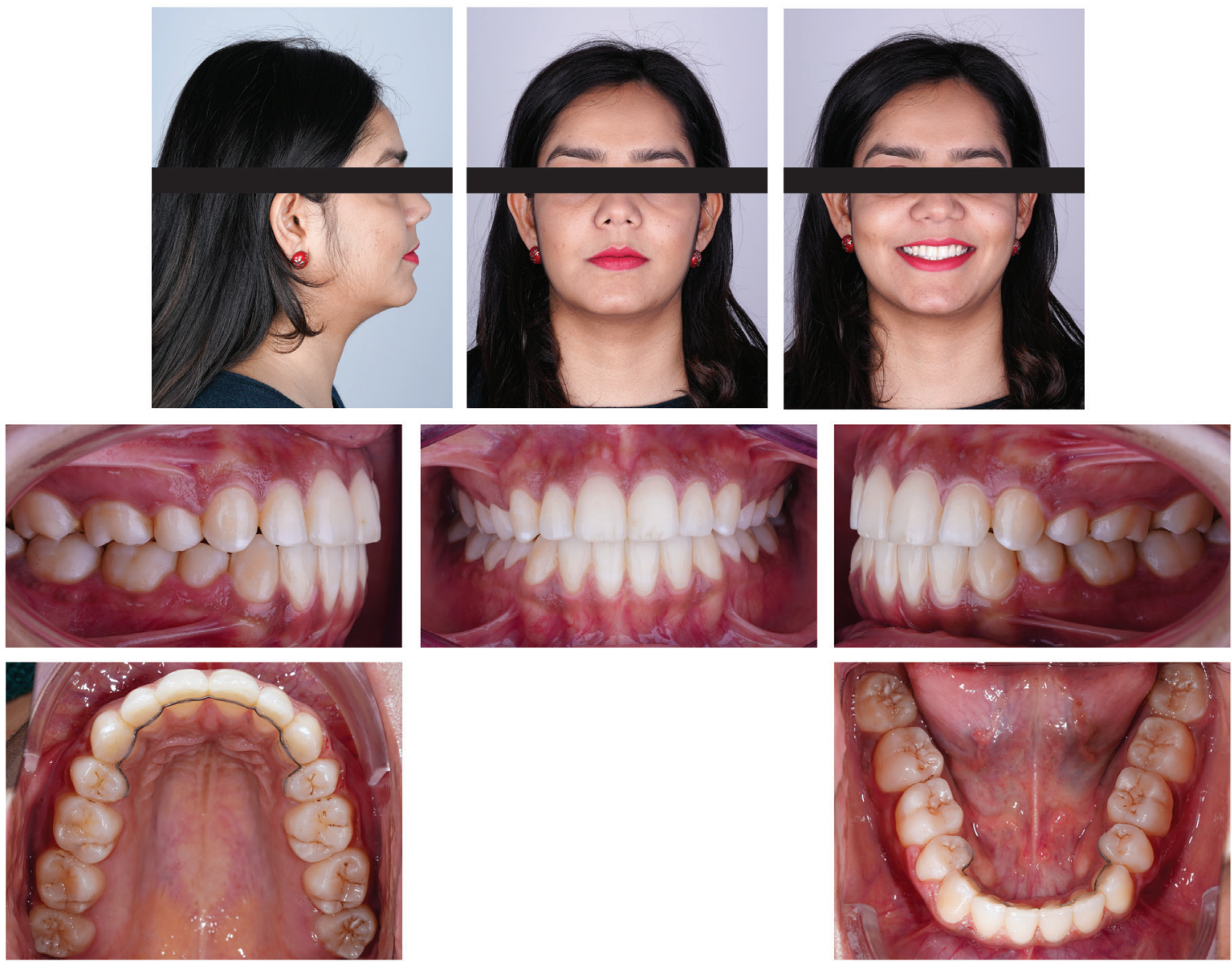

Figure 4: Posttreatment extra- and intra-oral photographs. Post treatment photographs demonstrate correction of the open bite, expansion of the maxilla and correction of the crossbites, correction of mandibular deviation, coinciding midlines, establishment of a Class I molar and canine occlusion, and ideal overjet and overbite. 


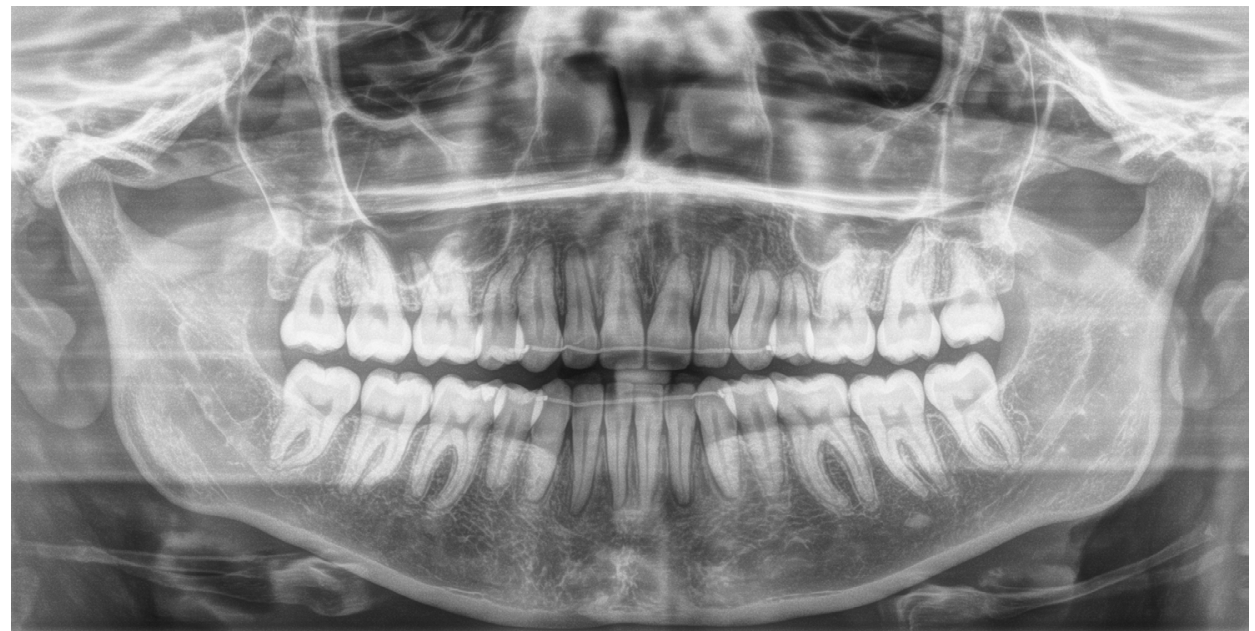

Figure 5: Posttreatment panoramic radiograph. Panoramic radiograph at the end of treatment shows good root parallelism and no increase in root resorption. The idiopathic sclerosis close to the root of the second left molar did not undergo any changes during the course of treatment.
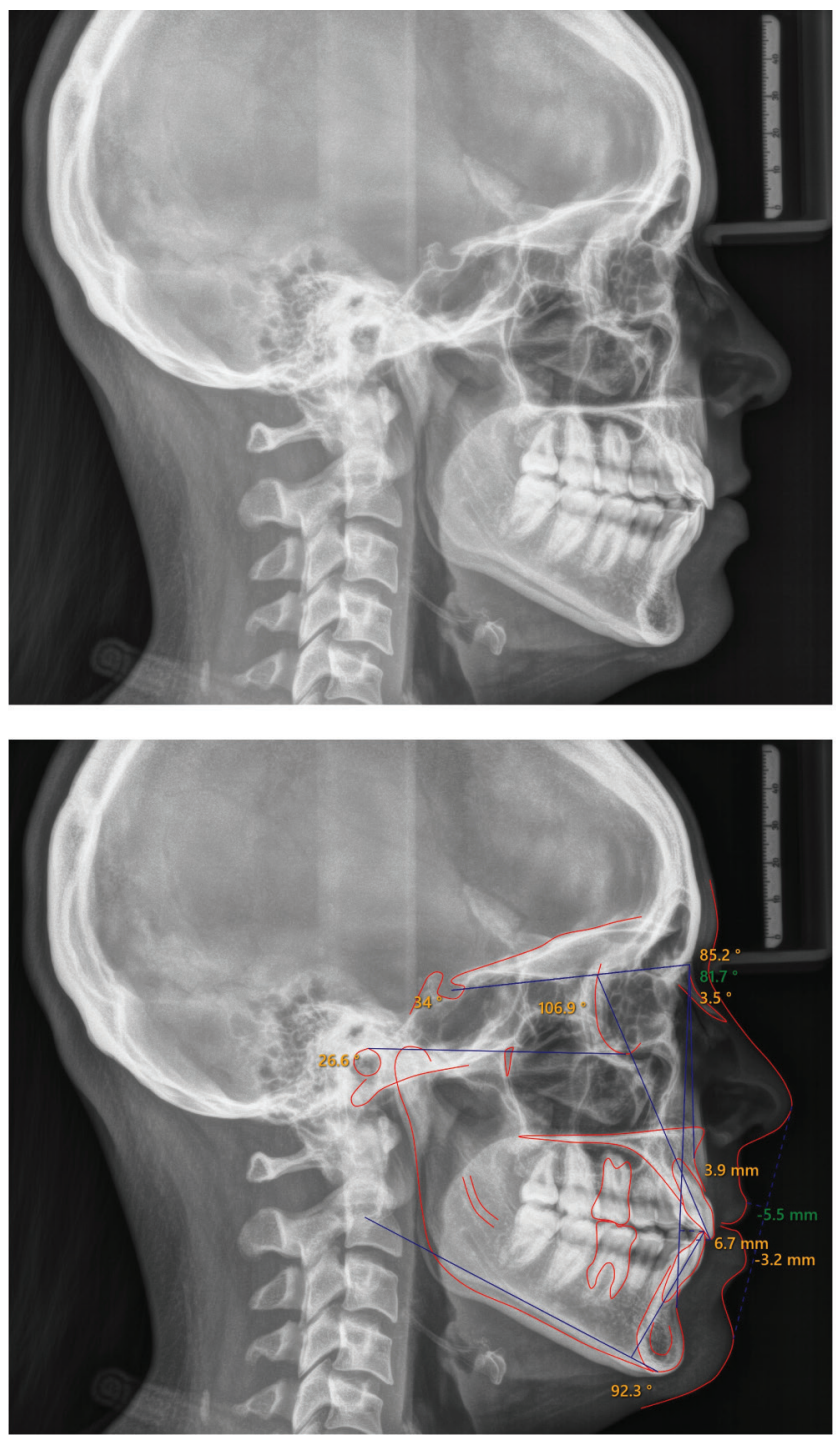

Figure 6: Posttreatment lateral cephalometric radiograph and analysis. Post-treatment cephalometric analysis shows correction of open bite, improvement in maxillary incisor inclination, overall improvement in relation between maxillary and mandibular dental arches and ideal overjet and overbite. 
A

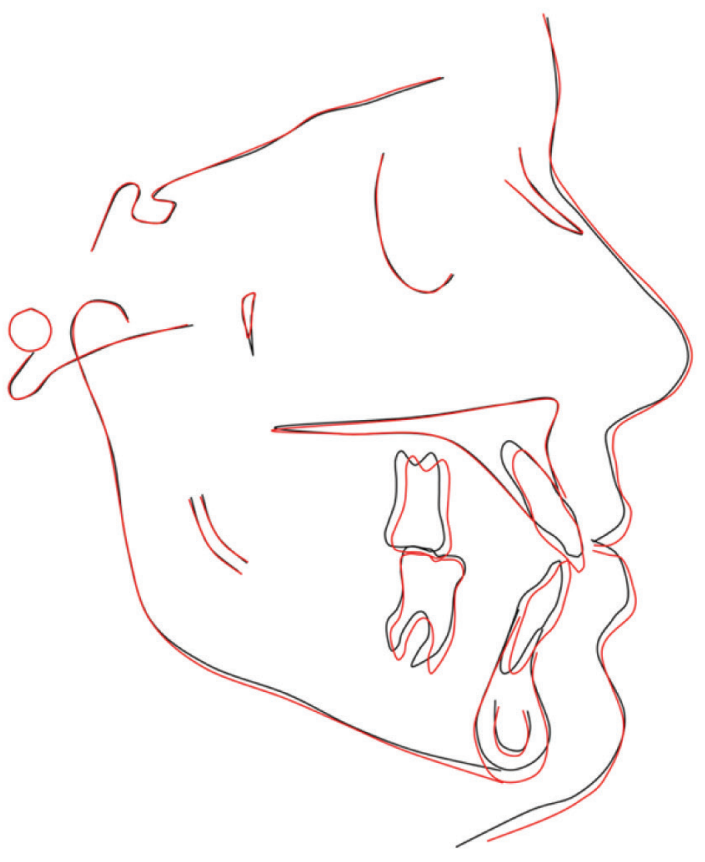

B
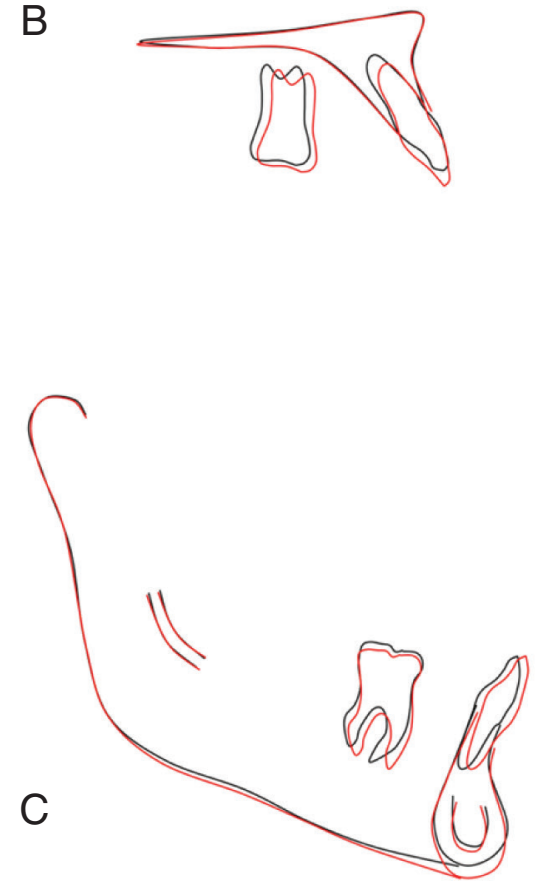

Figure 7: Superimposition of cephalometric analysis of pretreatment and posttreatment. Cephalometric superimposition on the anterior cranial-base demonstrates retroclinaiton of maxillary anterior teeth, extrusion of both maxillary and mandibular anterior teeth and flattening of the occlusal plane (A). Superimposition based on the body of maxilla, shows molar uprighting and incisor retroclination and extrusion (B). Superimposition based on inferior-alveolar nerve and inner profile of the mandibular symphysis demonstrates incisor extrusion and molar uprighting (C).

\section{Discussion}

With an increase in demand for faster, safer and more conservative treatment options, orthodontists must develop personalized treatment planning skills, to be able to deliver a mechanotherapy tailored to the patient's needs and preferences. These personalized treatment approaches should address both clinical and physiological demands of the patient, while engaging the patient in the selection of the treatment path as an active decision-making member of the treatment team. After all, it is our patients that have to adapt their life style based on the treatment approach that best matches their needs and desired outcome. The case presented here is an example of a personalized mechanotherapy approach, where the patient was educated about her treatment options and she actively participated in decision making, knowing the risks and demands of each step.

Major questions were raised due to the complexity of the case: First, should we provide treatment for a patient that presents with root resorption? Second, can we correct the open bite, knowing that the previous attempt failed? Third, can we expand the maxilla, knowing expansion in adults may hinder open bite correction? And lastly, but equally important for this patient, can we provide treatment in a reasonable time frame?

Considering the patient's occlusion and how it affected both function and esthetics, treatment could not be avoided even considering the risk of further root resorption. We decided to apply a very low force level, and monitor the patient with frequent panoramic radiographs to make sure further root resorption would not occur or at least would be minimized. As predicted, in response to careful mechanics, no further root resorption was observed in agreement with previous research showing that root resorption is mainly caused by excessive and uncontrolled mechanics [4].

Considering the malocclusion complexity, all treatment options including surgery, clear aligners and fixed appliances were reviewed, and the pros and cons of each approach discussed. The patient decided that comprehensive treatment with braces was an ideal and acceptable option. 
In this case, correction of open bite could not be based on uprighting of anterior teeth since no significant uprighting of anterior teeth was necessary and extraction of maxillary and mandibular premolars was not an option as premolars and space closure was completed during the patient's earlier orthodontics treatment. Since the mandibular plane angle and lower facial height were within normal limits, intrusion of posterior teeth to address the open bite was also not indicated. Therefore, the open bite was addressed mostly by extrusion of anterior teeth. Nevertheless, it was imperative to prevent further extrusion of posterior teeth, especially during expansion.

After expansion, posterior teeth tilt buccally, with the molar palatal cusps developing premature contacts with the opposing mandibular teeth. The use of temporary anchorage devices (TADs), allows application of intrusion forces palatally to control and/ or correct the extrusion caused by palatal expansion. Our patient refused the use of TADs and therefore, the intrusion force was applied utilizing a free object mechanical design in the form of a modified TPA [5]. Our end results demonstrate this technique was successful and no extrusion of posterior teeth was observed. Based on these mechanical options the presence of an open bite should not be considered a contraindication for palatal expansion.

While our mechanotherapy plan was able to maintain the height of the posterior teeth, it was necessary to extrude the maxillary anterior teeth to address the open bite. Segmental one-couple/two couple set ups were applied from the four maxillary incisors to the $1^{\text {st }}$ molar bands [6]. This enabled us to extrude the anterior teeth while at the same time applying an intrusion force on the posterior teeth.

It has been shown previously that non-surgical maxillary expansion in adults can be achieved without significant damage to periodontal tissues [7]. Similarly, our results show that application of slow expansion in an adult can correct the maxillary constriction. However, we cannot demonstrate how much of this correction was due to sutural expansion and how much was due to dentoalveolar changes since neither a CBCT scan nor anteroposterior radiograph was taken before, during or after treatment in this case. The absence of any signs of gingival recession or increase in buccal inclination of posterior teeth demonstrates that both skeletal and dental changes should be considered as the mechanism of expansion in this case. Expansion not only addressed the constricted arch but also provided space to resolve the crowding.

In open bite cases, the $3^{\text {rd }}$ molars usually are the first teeth to show premature contact, thereby worsening the open bite. While these teeth can be aligned with proper mechanics, this may significantly increase the treatment duration and the complexity of the mechanical design. Therefore, in open bite cases extraction of wisdom teeth can simplify the treatment and is generally recommended. In this case, the $3^{\text {rd }}$ molars not only were causing vertical problems, but they were also in full crossbite adding to the transverse problems. However, the patient refused $3^{\text {rd }}$ molar extraction. By including the $3^{\text {rd }}$ molars in the mechanotherapy plan from the beginning of treatment, the vertical and transverse problems associate with these teeth were kept under control.

The fact that treatment was accomplished in 10 months demonstrates that using a personalized mechanotherapy plan not only allowed us to achieve the treatment objectives without significant damage to supporting tissues, but to accomplish them in a short period of time.

It should be emphasized that the treatment in these cases is not over after appliances are removed, and retention is perhaps the most important phase of treatment. The etiologic factors that caused the crossbite and open bite are still present, therefore retainers should be designed to protect the occlusion, especially when the patient is sleeping and the muscular activity and rest position can interfere with occlusion. In the case presented here, the posttreatment stability was observed for only 8 months before the patient moved out of state. While short time follow up in this patient demonstrated excellent stability, the long-term observation by an orthodontist is required.

\section{Applied Innovation}

In an era of patient-centered care, the needs and preferences of our orthodontics patients should be carefully integrated into a detailed personalized mechanotherapy plan. The challenge becomes developing a customized and well-designed mechanotherapy plan that also takes into special consideration the mechanical and biological factors that can produce predictable results in a reasonable time frame. The patient needs to be well informed about risks, benefits and limitations of different mechanical designs, and be ready to accept some esthetic compromises while using different appliances. In this personalized approach to treatment, the educated patient becomes an active member of the decision-making team and routine "cookbook" orthodontics approaches need to be discarded. In this particular case, the mechanotherapy plan was customized to meet the patient's desires, without compromising the treatment outcome. To that end we used innovative mechanical designs such as one- and two- couple systems, free object designs and bite block therapy that addressed the patient's esthetics concerns and corrected the malocclusion without detrimental side effects, such as increasing root resorption or causing loss of periodontal support. 


\section{References}

1. de Freitas MR, Beltrão RT, Janson G, Henriques JF, Cançado RH. Long-term stability of anterior open bite extraction treatment in the permanent dentition. American Journal of Orthodontics and Dentofacial Orthopedics. 2004;125(1):78-87.

2. Bishara SE, Staley RN. Maxillary expansion: clinical implications. American Journal of Orthodontics and Dentofacial Orthopedics. 1987;91(1):3-14.

3. Killiany DM. Root resorption caused by orthodontic treatment: an evidence-based review of literature. Semin Orthod. 1999;5(2):128-33.

4. Viecilli RF, Kar-Kuri MH, Varriale J, Budiman A, Janal M. Effects of initial stresses and time on orthodontic external root resorption. Journal of Dental Research. 2013;92(4):346-51.

5. Alikhani M. Mechanotherapy in Orthodonitcs Volume II. CTOR Press, Hoboken NJ; 2021.

6. Alikhani M. Mechanotherapy in Orthodontics Volume I. CTOR Press, Hoboken NJ; 2018.

7. Handelman CS, Balakrishnan M, BeGole EA, Viana GC. Bimaxillary transverse constrictionin adults: short-term follow-up of non-surgical arch expansion. Orthod Craniofac Res. 2020 May;23(2):202-209. 